\title{
USE OF MICRO-ELECTRO MECHANICAL SENSORS IN BEARING CONDITION MONITORING: A REVIEW
}

\author{
H. S. KUMAR \\ Department of Mechanical Engineering, NMAM Institute of Technology, Nitte, Visvesvaraya Technological University, \\ Belagavi, Karnataka, 574110, India
}

\begin{abstract}
The rotary machines are widely used in industries and their maintenance is vital, as failures leads to increase in down time, lower production and profit to the industries. The rotary machines in industries have always been an area, where there is a scope to explore more and to achieve next gen-technology remains a challenge. In this regard, Micro-Electro Mechanical Systems (MEMS)-sensors are gaining wider acceptance and adoption for static and dynamic applications. Recent increase in demands for reliable Condition Monitoring (CM) has necessitated seeking alternatives to expensive conventional accelerometers to perform multi control and monitoring tasks. Owed to their size and cost, MEMS accelerometers are one of the alternative options. This review paper aims at discussing important MEMS-based sensors use in the rotary machine fault diagnosis.

KEYWORDS: Bearing, Condition, Monitoring, MEMS Sensor
\end{abstract}

Received: Jun 08, 2020; Accepted: Jun 28, 2020; Published: Sep 09, 2020; Paper Id.: IJMPERDJUN20201124

\section{INTRODUCTION}

Recently, machine condition monitoring (MCM) is becoming increasingly successful and advanced. The CM is performed in two ways: continuous or periodic monitoring of machine vibrations. Vibration transducers are mainly used to transform vibrations in to an electrical signal. Accelerometers are the most popular general purpose vibration transducers. Different technologies are used in constructing the accelerometers the industry relies on the use of piezoelectric accelerometers as it offers following merits: compact, rugged, self generating and wide dynamic range. The accelerometers cost is too high if its sensitivity is also high [1-2]. Many researchers shown that vibration based condition monitoring method provides better and more solid data, thereby resulting in a more compelling condition monitoring program with huge cost benefits to industries [3]. Rolling element bearings are most commonly used in rotary machine right across the industries. Due to varying compliances, the bearing acts as vibration and noise sources as much as defects present on them. The defects may be categorised into distributed and localizes defects. The defects may be due to manufacturing errors, improper installation, lubrications or even ear or fatigue [4-5]. Usually, REB has four essential components: inner race (IR), outer race (OR), rollers, and cage or separator as shown in fig.1. The inner race, outer race, and rollers holds the load acting on the bearing and rolling elements are separated by cage thereby avoiding friction between them. Rolling elements such as balls, cylindrical, tapered, needles, or barrel type rollers enclosed in a cage that allows equal separation and helps in avoiding internal friction. Bearing defects are often a danger bell of other machinery faults since, bearing defects may lead to for example, misalignment and/or imbalance of shafts. Therefore incipient identification of bearing failure is vital to get rid of the system failure [4]. 


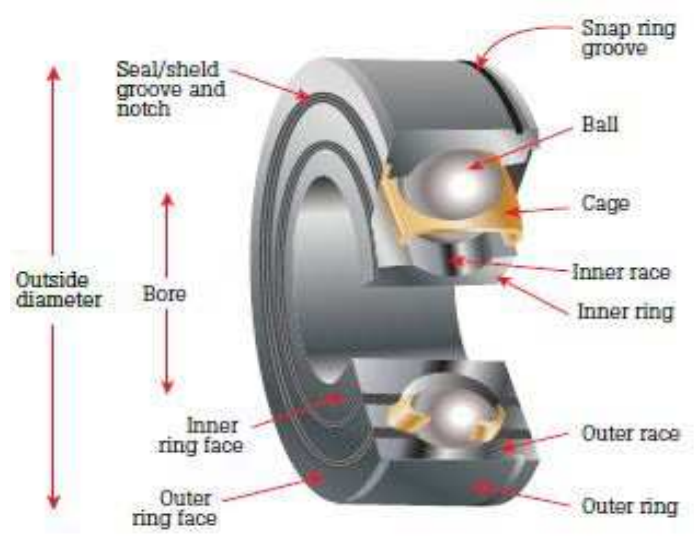

Figure 1: Parts of Rolling Element Bearing [4].

Micro electro mechanical system (MEMS) accelerometers are the most trending and useful vibration sensors. The MEMS are manufactured in such a way that, the signal conditioning and the sensors are incorporated in a solitary chip at a little cost with great accuracy, performance, and excellent reliability. MEMS accelerometers utilize different methods for estimating forces like strain gauge, silicon capacitive, silicon piezoresistive, micro machined resonators, and force balance. Among these sensing methods, the capacitive sensing method has lately turned into attractive since its noise performance is low, good DC response, insensitivity towards temperature variations, low power consumption, high sensitivity, and a basic setup [6-7].

A MEMS accelerometer detects vibration with assistance of micro-fingers in relative proximity to fixed anodes. At a point when the system is exposed to vibrations the movable fingers are averted and the separation amongst fingers and the electrodes changes. This produces a capacitance change among the fingers and the electrodes. The distance and capacitance change can be related with the following equation [6-8]:

$$
C=\epsilon_{0} \epsilon_{\tau}\left(\frac{A}{d}\right)
$$

Where $\mathrm{C}$ is the capacitance $\mathrm{n}$ farads, $\mathrm{F}, \varepsilon_{0}$ is the permittivity of free space in $\mathrm{F} / \mathrm{m}, \varepsilon_{\mathrm{r}}$ is the relative permittivity or dielectric constant of the insulator used, A is the area of each electrode in $\mathrm{m}^{2}, \mathrm{~d}$ is the diameter in $\mathrm{m}$. The capacitance change is converted to a output voltage variation. The fixed plates are driven by $180^{\circ}$ out-of-phase square waves. Acceleration deflects the beam and unbalances the differential capacitor, resulting in an output square wave whose amplitude is proportional to acceleration. A continuous analogous signal is produced using this principle for the indication of the sensor output. If the sensor is at constant velocity, there will be no acceleration, the signal will be constant [6,].

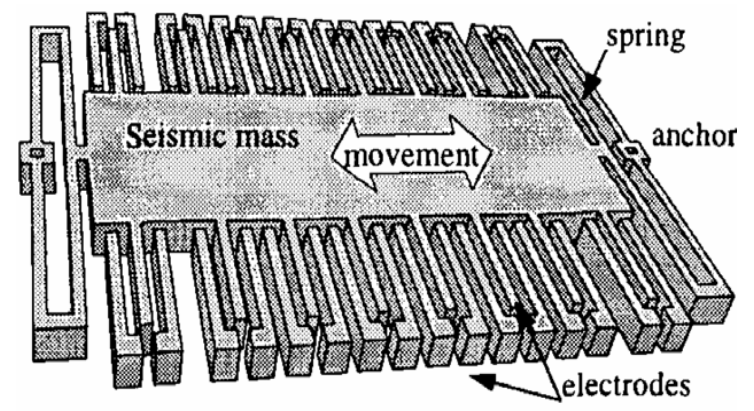

Figure 2: MEMS Vibration Sensor [6]. 
But when the accelerometer is mounted on a vibrating body, it produces a continuous but varying acceleration; the output signal will be corresponding to the vibration of the body. Figure 2 demonstrates a photo of a MEMS accelerometer showing the deflecting fingers and fixed electrodes. This only shows acceleration in one axis. To get acceleration in two or three directions, separate sensors can be used. The MEMS is an essentially analogue device, hence to get digital output it requires an Analog to Digital Converter (ADC) [6-8].

\section{MEMS TECHNOLOGY}

MEMS is a rising research field. Here, the membrane or cantilever type mechanical components are manufactured at a more similar scale to that of microelectronic circuits. It come into sight that these gadgets share the components of size below $150 \mu \mathrm{m}$ and these components are machined using other techniques globally called micro-fabrication technology rather using standard machining. Unlike electronic circuits which are essentially compact and solid structures, MEMS have cavity, holes, membranes, channels, cantilevers, etc. A broad knowledge on silicon and silicon based micro fabrication captured by decades of research in microelectronics resulted in importance on MEMS based on silicon chips. At a point when MEMS are based on silicon, microelectronics process should be modified to serve for deep layer deposition, thicker etching and to find special techniques which can free the mechanical structures. MEMS require a totally unique set of art, it requires deep knowledge on electronics, mechanical and material science assumes a basic role. Then, MEMS can be manufactured not only on silicon but also on polymer, glass, quartz or metals [6]. The structures with micrometric features are manufactured using micro-fabrication technique. Conventional manufacturing techniques are not suitable for MEMS fabrication. Methods such as photolithography, thin film deposition by using chemical vapour deposition (CVD) or physical vapour deposition (PVD), thin film growth by using oxidation and epitaxy, doping by ion diffusion, wet etching, dry etching, etc have all been embraced by the MEMS technologists.

Like microelectronics, the selection of good material for MEMS is not based on properties like carrier mobility, but it is more dependent on mechanical aspect such as low or controllable internal stress, similarity with other materials, low handling temperature, and thick layer patterning probabilities. The perfect mechanical material, silicon and its compounds, such as silicon (di) oxide $\left(\mathrm{SiO}_{2}\right)$ and silicon nitride $\left(S z_{x} N_{y}\right)$ are mainly used for MEMS fabrication. Silicon is lighter and is stronger than steel. Also it has high critical stress and is a perfect crystalline; hence it has got no elastic limit at ideal working conditions. But its brittleness may cause difficulty in wafer holding, although it hardly fails in MEMS. Silicon also has a high coefficient of piezo-resistance, which helps in increasing the sensitivity. Silicon nitride is another material used in MEMS, is considerably harder than silicon. It can be placed in fine layer to produce $0.99 \mu m$. Another derivative silicon carbide ( $\mathrm{SiC}$ ) is also used in MEMS. Some of the materials other than silicon and its derivatives, such as, quartz, polymer, metals, and glass are also used in MEMS fabrication [4-7].

\section{MEMS SENSOR USE IN BEARING FAULT DIAGNOSIS}

Bearing is the most important component in any rotary machines, but also it is common failure unit. An effective condition monitoring of the bearing can effectively prevent costly, even catastrophic downtime. Many researchers have been working on the use of MEMS in CM of rotary machines. MEMS sensors provide same results when compared to conventional sensors and is found in the following reviews. Albarbar et al. (2008) studied performance of three dissimilar types of accelerometers and compared the results with commercial accelerometers. The experiments were performed on $\mathrm{CNC}$ machines in prototypical industrial environment for various excitations including impulse, sinusoidal, 
and random. They compared the calculated responses of the MEMS accelerometers with the commercial ICP accelerometer. Although they found a very small shift in phase, the MEMS accelerometer showed good result for the random and sinusoidal measurements [8]. Son et al. (2009) developed a cost-effective maintenance technique to acquire three types of signal from induction motor. They presented a novel smart sensor system for induction motor fault diagnosis that can replace expensive conventional accelerometers. Algorithms like Support Vector Machine (SVM), Random forest (RF) and linear discriminant analysis were applied to classify the fault in the motor. The smart sensor was shown good performance in machinery fault diagnosis [9]. Albarbar et al. (2017) gave insight into outline, working principles and practical guidance of MEMS accelerometers. Details of the experimental set up, signal condition and data processing were advanced to construct simple analyzing system. The performance of the system using sinusoidal, impulse and random excitation were evaluated. Parallelly, the frequency response function, Signal to Noise Ratio (SNR), phase distribution and comments on them were tabulated. The result showed that the MEMS accelerometers offer excellent alternatives to conventional type [10].

\section{Use of MEMS Sensors in Bearing Fault Diagnosis}

This section covers the role of MEMS sensor in bearing fault diagnosis.

Elandy et al. (2013) proposed an On-shaft wireless condition monitoring measurement system for vibration based condition monitoring of machines. It has given a clear picture of measurement scheme and initial observations made on a test rig that is used in the experiment. It has been observed that the measured accelerometer consists of DC and AC components. The DC component of the measured acceleration directly indicated rotor unbalance [11]. Maruthi.G.S et al. (2014) employed motor vibration and current signals to identify fault in inner race of the bearing of small HP induction motor. They carried out experimental investigations under unbalance voltage, no load, and full load conditions. They investigated the outcome of inner race bearing fault on the air gap length modulation. From the results it can be seen that, as the motor load increases, fault magnitude in vibration decreases and current signature increases [12]. Chaudhury et al. (2014) integrated the basic sensor \& intelligent vibration analysis to develop a cost effective MEMS based vibration analysis system. This system can easily be used for vibration monitoring of various rotary machines. A $7.5 \mathrm{KW}, 3 \varphi, 440 \mathrm{~V}$, 4 pole induction motor was used to check the sensitivity of the sensor, effectiveness of the proposed system in signal processing and diagnosis. The experiments were carried out to inspect the capability of the system to obtain the fault frequency peaks under various fault combinations. Also they have used an advanced noise cancellation technique to enhance the signal detection capability in the acceleration signal. These methods found to be very successful for filtering the periodic, random and white Gaussian noise in real-time acceleration signals [13]. Uhlmann et al. (2015) presented the concept of a wireless sensor network (WSN) using MEMS sensors and Raspberry Pi 2 as DAQ, signal processing and classification. The use of these methods for CM applications and selected algorithms were introduced. Also they showed that this technique can be used to detect faults in rotating components in production system. A clear picture of industrial application of this concept was shown and visualization of results were achieved. The concept presented was implemented to enable embedded data acquisition and signal processing. They concluded that this concept can be configured to variety of industrial applications to realize condition monitoring of components [14]. Feldman et al. (2016) demonstrated MEMS technology to achieve a vibration based condition monitoring, diagnostics, and characterization of a radial air turbine supported under micro ball bearing. By using a software suite and sensor network they developed a multimodal software suite for platform automation and monitoring the sensor. They used MEMS sensor suit to monitor and detect several 
functional parameters favorably in time and frequency domain such as wobble, rotor imbalance, etc. From the results it observed that the data obtained from the system have helped for the prompt identification of failure and fault diagnosis system [15]. Son et al. (2016) used MEMS-based accelerometers and current sensors for fault identification of motor. The vibration and current signals are captured to prove the reliability of the MEMS-based sensors. They estimated diagnostic potential of the induction motor fault using MEMS accelerometer. They concluded that the cost effective MEMS accelerometers gave comparatively same result as the conventional accelerometers [16]. Elandy et al.(2014) presented Onshaft Vibration measurement (OSVM) technique and its applicability to diagnose different faults introduced in an experimental test rigusing MEMS sensor, designed to model a rotary machine. They compared the fault diagnosis performance of OBVM and OSVM. The comparative results revealed the superiority of OSVM, as it resulted in higher accuracy and was able to separate similar faults in comparison to OBVM [17]. Nembhard, A. D., et al.(2013) developed a simplified fault diagnostic method, which used single vibration and temperature sensor on each bearing. Experimental results revealed that combination of vibration and temperature sensor resulted in better separation of healthy and defective conditions, when compared to use of vibration sensor alone. The proposed method was simple and non-intrusive and the approach can be tested using different test rigs with different faults for validating the usefulness of the proposed method [18]. Konforty, Shlomi, et al.(2016) presented an improved bearing sensing diagnostic approach. By mounting miniature sensors in the vicinity of the bearing, there was an improvement in the signal-to-noise ratio of the bearing vibration signals and transmission path concerns were addressed. They carried out fault diagnosis in two ways: Placing a MEMS accelerometer on the bearing outer race and the measurement of strain using an optical fibre on the bearing housing. The two methods provided better fault detection, whenever the sensor was closer to the defective bearing. A number of tests were conducted to understand the influence of different factors involved, when designing the sensors. A similar response was found for the two methods [19]. Alaa Abdulhady Jaber et al. (2014) presented a detailed review on various techniques used in condition monitoring of machines and pointed that vibration and acoustic emission techniques are widely used to monitor industrial machines. The features extracted from the acquired signal need to be analyzed which is an issue. Majority of researchers have come out with new features and feature selection techniques for fault diagnosis. In simple terms, to design a reliable condition monitoring technique, correct sensors have to be selected in order to get accurate signals from faulty parts, and appropriate signal analysis techniques have to be used, since these have a significant impact on the sensitivity of the features extracted from the signals captured [20]. Han and Kim et al.(2016) proposed automated approach for bearing fault diagnosis based on the two dimensional analysis of vibration signals under variable speed conditions. For a defective bearing vibration signal, the texture of the image is different from that of the image texture of the normal bearing and displays minimum variation with the shaft speeds. Micro texture analysis of these images will provide distinctive fault signature for each defective condition, which can be used to pin point defects at variable speeds. The fault signatures are fed to K-nearest classifier to classify different conditions. The proposed approach is verified using bearing fault dataset of Case Western Reserve University (CWRU), and the results are compared with those of a spectrum imaging-based approach [21]. Subimal et al.(2014) designed and developed a low cost MEMS sensor system. They developed a new noise cancellation algorithm using auto-correlation function and adoptive threshold based filters to enhance signal characteristics. The proposed method was assessed under different operating and fault conditions, in order to extract fault features of the weak fault signals immersed in the noise. The proposed method was found to be effective for filtering the periodic, white Gaussian and random noise in real-time acceleration signals [22]. Kavitha $\boldsymbol{\&}$ Thangadurai (2014) presented advance detection of faults in drives using MEMS for Industrial applications. Proper condition monitoring leads to improve the reliability of the machine and leads to better machine efficiency, reduced 
downtime by eliminating unexpected mechanical or electrical failures. In this work, the MEMS technology was used to detect the false in advance by vibration monitoring method. The severity level of abnormality and the remaining usable life were also investigated [23]. Prashanth et al. (2018) compared the performance of the conventional and MEMS accelerometer using a customised bearing test rig. The vibration signals for three conditions namely normal bearing $(\mathrm{N})$, defect on inner race (IR) and defect on outer race (OR) were acquired and FFT analysis revealed similar frequency responses from both conventional and MEMS accelerometer. Also, fault diagnosis capability of MEMS accelerometers had been tested using classifiers namely Artificial Neural Network (ANN) and K- Nearest neighbourhood (KNN). The results revealed that conventional accelerometers can be replaced by MEMS accelerometers for low cost condition monitoring [24].

\section{CONCLUSIONS}

There has been significant development and improvements in the use of MEMS sensors in fault diagnosis. The rapid increase in the use is attributed to compactness, low cost and efficient results in fault diagnosis. The literature review presented mainly focussed on the use of MEMS sensors in rotary machine fault diagnosis.

\section{REFERENCES}

1. Pandiyan, J., Umapathy, M., Balachandar, S., Arumugam, M., Ramasamy, S., \& Gajjar, N. C. "Design of industrial vibration transmitter using MEMS accelerometer". Journal of Physics: Conference Series Vol. 34, No. 1, (2006), 442447 .

2. Patil, M. S., Jose Mathew, and P. K. RajendraKumar. "Bearing signature analysis as a medium for fault detection: A review." Journal of Tribology 130.1 (2008), 014001-1 to 014001-7.

3. Peng, Z. K., and F. L. Chu. "Application of the wavelet transform in machine condition monitoring and fault diagnostics: a review with bibliography." Mechanical systems and signal processing 18.2 (2004): 199-221.

4. Mechefske, C.K, "Machine condition monitoring and fault diagnosis", Vibration \& shock handbook, CRC press, Taylor and Francis group, Mechanical engineer divisor, chap25, 2005.

5. Jarno Junnola, "The suitability of low-cost measurement systems for rolling element bearing vibration monitoring", PhD Thesis, 2007.

6. Matej Andrejaši`c, "Mems accelerometers", PhD thesis, University of Ljubljana, 2008.

7. Jyothi K Sinha, "On standardisation of calibration procedure for accelerometer", "Journal of Sound and Vibration 286", 2005, pp417-427.

8. Alhussein Albarbar, Samir Mekid, Andrew Starr, Robert Pietruszkiewicz, "Suitability of MEMS Accelerometers for Condition Monitoring: An experimental study", Sensors 8, 2008, 784-799.

9. Jong-Duk Son, Gang Niu, Bo-Suk Yang, Don-Ha Hwang, Dong-Sik Kang, "Development of smart sensors system for machine fault diagnosis", "Expert Systems with Applications 36" 2009, pp 11981-11991.

10. A. Albarbar and S.H. Teay, "MEMS Accelerometers: Testing and Practical Approach for Smart Sensing and Machinery Diagnostics", "Microsystems and Nanosystems", 978, pp319-324.

11. M. E. Elandy, J.K.Sinha, S.O.Oyadiji, "On-shaft wireless vibration measurement for condition monitoring of rotating machine", The University of Manchester, Manchester M13 9PL, 2013. 
12. Maruthi G. S, Vishwanath Hegde, "Preliminary Investigation on Bearing Fault Analysis in Three Phase Induction Motor by MEMS Accelerometer", "Preliminary Investigation on Bearing Fault Analysis in Three Phase Induction Motor by MEMS Accelerometer”, 2014, 4799-5496.

13. Subimal Bikash Chaudhury, Mainak Sengupta, Kaushik Mukherjee, "Vibration Monitoring of Rotating Machines Using MEMS Accelerometer”, International Journal of Scientific Engineering and Research, Volume 2 Issue 9, 2014.

14. E. Uhlmann, A. Laghmouchi, E. Hohwieler, C. Geisert, "Condition monitoring in the cloud", "Procedia CIRP 38 ", 2015, pp53-57.

15. Jeremy Feldman, Brendan Michael Hanrahan, Saswat Misra, Xiao Zhu Fan, Christopher Mike Waits, Member, Paul D. Mitcheson, Senior Member, Reza Ghodssi, Fellow, "Vibration-Based Diagnostics for Rotary MEMS", "Journal of Microelectromechanical System, Vol. 24, No. 2, 2015, 289-293.

16. Jong-Duk Son, Byung-Hyun Ahn, Jeong-Min Ha, Byeong-Keun Choi, “An availability of MEMS-based accelerometers and current sensors in machinery fault diagnosis”, Measurement 94, 2016, pp680-691.

17. Elnady, M. E., Jyoti K. Sinha, and S. O. Oyadiji. "Identification of critical speeds of rotating machines using on-shaft wireless vibration measurement." Journal of Physics: Conference Series. Vol. 364. No. 1. IOP Publishing, 2012, pp.110.

18. Nembhard, A. D., Sinha, J. K., Pinkerton, A. J., \& Elbhbah, K., Fault diagnosis of rotating machines using vibration and bearing temperature measurements. Diagnostyka, 14(3), 2013, pp. 45-51.

19. Konforty, S., Khmelnitsky, M., Kressel, I., Klein, R., Tur, M., \& Bortman, J. Bearing health monitoring using optical fiber sensors, European conference of Prognostics \& Health management society, 2016, pp. 1-7.

20. Jaber, A. A., \& Bicker, R., The state of the art in research into the condition monitoring of industrial machinery. Int. J. of Current Engineering and Technology, 4(3), 2014, pp. 1986-2001.

21. Jaber, A. A., \& Bicker, R., Design of a Wireless Sensor Node for Vibration Monitoring of Industrial Machinery. International Journal of Electrical and Computer Engineering (IJECE), 6(2), 2016, pp. 639-653.

22. Subimal, B., Mainak, S., \& Kaushik, M., Vibration Monitoring of Rotating Machines Using MEMS Accelerometer. International Journal of Scientific Engineering and Research (IJSER), 2(9), 2014, pp. 2347-3878.

23. Kavitha V, R Thangadurai,(2014), Advance Detection of Faults in Drives Using MEMS, IJAREEIE , Vol.3 (4), pp. 203208

24. Prashanth B, Kumar H. S, Srinivasa Pai P, Muralidhara, Use of MEMS based Vibration Data for REB Fault Diagnosis, International Journal of engineering \& Technology, 7 (3.34), (2018), pp. 714-718

25. D. L. Gunapala, O. Gunapala \& S. Egodage, "Application of Modeling and Simulation Tools for Design of Plastic Products “, International Journal of General Engineering and Technology (IJGET), Vol. 8, Issue 1,pp. 1-8

26. D K Shukla, Mahendra Singh \& K K Jain, "Bearing Capacity of Footing on Slopping Anisotropic Rock Mass “, IMPACT: International Journal of Research in Engineering \& Technology (IMPACT: IJRET), Vol. 2, Issue 4, pp. $217-$ 232

27. R. K. Dhal, "Vorticity on MHD Free Convection Flow of Fluid with Heat Transfer through Porous Medium by an Oscillating Porous Plate in Slip Flow Region “, BEST: International Journal of Humanities, Arts, Medicine and Sciences (BEST: IJHAMS), Vol. 3, Issue 7, pp. 77-82 
28. N. Anusha \& P. Sai Avinash, "Experimental Investigation on Flexible Pavements with Various Soil “, International Journal of Civil Engineering ( IJCE), Vol. 6, Issue 6,pp. 61-72 